\title{
A MULTI-WAVELENGTH ANALYSIS OF THE MATTER DISTRIBUTION IN CLUSTERS OF GALAXIES
}

\author{
HAIDA LIANG \\ CEA/DSM/DAPNIA Service d'Astrophysique \\ F-91191 Gif sur Yvette, Cedex, France
}

\begin{abstract}
We combine the X-ray data and the Sunyaev-Zel'dovich effect with the constraints from gravitational lensing for an intermediate redshift cluster to check the consistency of various mass estimates and the validity of the assumption of hydrostatic equilibrium, and to deduce the properties of the intra-cluster gas, the profile of the cluster potential and $H_{o}$.
\end{abstract}

\section{Introduction}

Recent developments in the field of gravitational lensing have made detailed and independent analyses of the mass distribution of clusters of galaxies possible. The traditional tracers of the cluster potential are the cluster galaxies and the intracluster gas. By assuming that the cluster is in virial equilibrium, the observed velocity dispersion of the member galaxies can be used to deduce the cluster mass. The intracluster gas is observed either through their thermal X-ray emission or the Sunyaev-Zel'dovich effect (SZ effect; Syunyeav and Zel'dovich 1981). The cluster potential can then be deduced from the X-ray surface brightness and temperature and/or the Sunyaev-Zel'dovich effect by assuming that the gas is in hydrostatic equilibrium. Unlike these two methods, gravitational lensing effects are independent of the dynamical state of the cluster since the distortions of the background galaxies only depends on the gravitational field of the cluster. It has been shown that the position of a giant arc constrains very well the mass interior to the radius of the arc (Kochanek 1992), and at the same time the weak distortion of the background galaxies provides a good estimate of the shape of the cluster potential in the outer parts of the cluster (Kaiser and Squires 1993). In this paper we will combine the various in- 
dependent methods of cluster mass analysis for a medium redshift cluster. We will check the validity of the assumption of hydrostatic equilibrium and then combine the lensing constraints with the X-ray data and the SZ effect to constrain the cluster potential and deduce $H_{0}$.

\section{A Multi-wavelength Analysis of A2218}

A2218 is an Abell richness class 4 cluster at a redshift of $\mathrm{z}=0.175$ with a velocity dispersion of $1370_{-120}^{+160} \mathrm{kms}^{-1}$ (Le Borgne et al. 1992). There has been a large amount of data collected for the cluster in the optical, Xray and radio wavelengths by various groups. It has a X-ray luminosity of $L_{x}=1.01 \times 10^{45} \mathrm{ergs} / \mathrm{s}$ in the $2-10 \mathrm{keV}$ energy range and a temperature of $8 \times 10^{7} \mathrm{~K}$ measured by the Ginga satellite ${ }^{1}$ (McHardy et al. 1990, David et al. 1993). There are gravitational lensing arcs found in this cluster, both around the central $\mathrm{cD}$ and the bright galaxy of a smaller clump (Pello et al. 1992). Furthermore, A2218 is one of the few clusters where the SunyaevZel'dovich decrement has been successfully measured (Jones et al. 1993; Figure 3). Given the wealth of data available on this cluster, we can obtain a better understanding of the dynamical state and/or properties of the intra-cluster medium, as well as the matter distribution and $H_{0}$ than most other clusters available.

Both the X-ray surface brightness distribution and the SZ effect can be linked to the cluster gravitational potential via the equation of hydrostatic equilibrium. If the intracluster gas is in hydrostatic equilibrium then the gas density is given by $\rho_{g} / \rho_{0}=\exp \left(-\frac{\mu m_{p}}{k T_{g}}\left[\phi(r)-\phi_{0}\right]\right)$, where we have also assumed that the gas is isothermal for simplicity. Thus the X-ray surface brightness is given by

$$
S_{x}(R) \propto \int_{R}^{r_{t}} \exp \left(-\frac{2 \mu m_{p}}{k T_{g}}\left[\phi(r)-\phi_{0}\right]\right) \Lambda\left(T_{g}\right) \frac{r d r}{\sqrt{r^{2}-R^{2}}}
$$

and the SZ effect is given by

$$
\Delta T_{r}(R) \propto \int_{R}^{r_{t}} \exp \left(-\frac{\mu m_{p}}{k T_{g}}\left[\phi(r)-\phi_{0}\right]\right) T_{g} \frac{r d r}{\sqrt{r^{2}-R^{2}}}
$$

where $\phi$ is the cluster potential, $T_{g}$ is the gas temperature, $\Lambda\left(T_{g}\right)$ is the temperature dependent emissivity. The cluster potential $\phi$ is constrained by the amount of shear produced on the background galaxies by the cluster. In the case where there is a giant arc at the center of the cluster, the projected mass within the arc is well determined and thus the central parts

\footnotetext{
${ }^{1}$ Preliminary results from ASCA also show that the gas temperature is $\sim 6.8 \mathrm{keV}$ and that the gas clearly extends to 4 Mpc (M. Bautz 1994)
} 


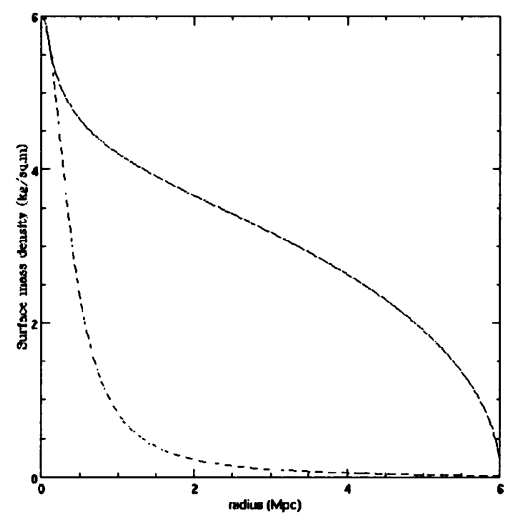

Figure 1. The cluster surface mass density in units of $\mathrm{kg} / \mathrm{m}^{2}$. The solid curve gives the surface mass density corresponding to the potential that fits all the X-ray and SZ effect data. The dashed curve gives the surface density profile corresponding to $\left[1+\left(r / r_{c}\right)^{2}\right]^{-1}$ but normalized at the arc radius to fit the constraint imposed by the arc. This functional form was found to fit the galaxy number density distribution (Dressler 1976).

of the potential is constrained through Poisson's equation. In a spherically symmetric cluster, the position of the giant arc corresponds to the locus of the critical circle. The mean surface density inside the critical circle of radius $\mathrm{b}$ is given by the critical surface density (Blandford \& Kochanek 1988), i.e.

$$
\Sigma(b)=\Sigma_{c r i t}=\frac{c^{2}}{4 \pi G} \frac{D_{s}}{D_{l} D_{l s}}
$$

where $D_{s}, D_{l}$ and $D_{l s}$ are the distance to the source, to the lens and the distance between the lens and the source. In the case of A2218, one of the large arcs (object number 359 in Pello et al. 1992; see also Kneib et al. this proceeding) has a spectroscopic redshift of $z=0.702$. As pointed out in Miralda-Escudé \& Babul (1995; MB here after), there is another object in the field (object number 328 in Le Borgne et al. 1992) which is very likely another image of the arc (number 359 ). The critical line should thus intersect the arc which puts the critical radius at $20.8^{\prime \prime}$ (or $0.08 \mathrm{Mpc}$ ).

Our aim here is to see if we can find a potential profile that would fit the strong lensing constraint, X-ray data and the SZ effect. In order to compare with the observed data we need to simulate both the X-ray and radio observations. In the case of the X-ray data we need to convolve the ROSAT PSF with the X-ray surface brightness deduced from equation (1) (see Figure 2). Similarly, we multiply the SZ radio surface brightness deduced from equation (2) by the primary beam of the Ryle telescope and Fourier transform the resulting profile to obtain the SZ flux density expected at various baseline lengths of the interferometer (see Figure 3). The 


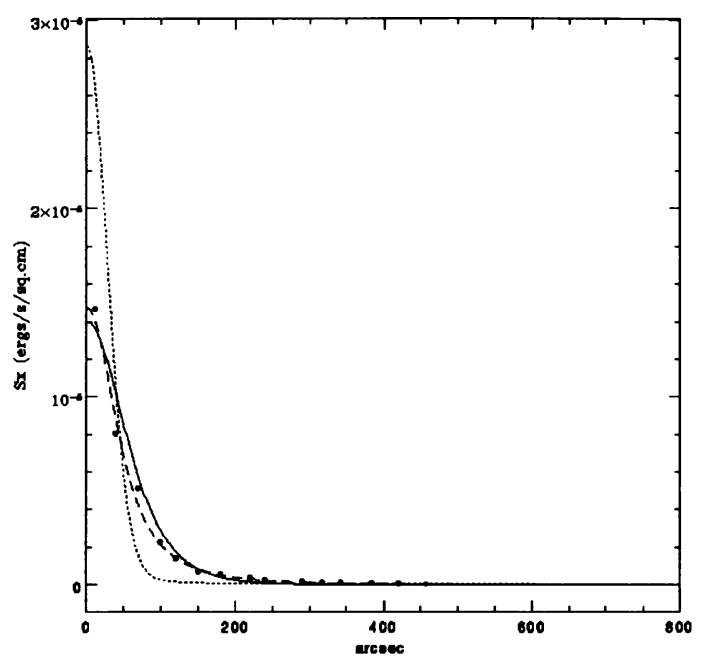

Figure 2. X-ray surface brightness profile. The filled circle is the observed surface brightness from ROSAT PSPC (MB). The solid curve shows the simulated X-ray surface brightness for a cluster potential given in Figure 1 with $T_{g}=8 \times 10^{7} \mathrm{~K}$. The dotted curve corresponds to the best fit for the cluster density profile of Model 1 given in MB for $T_{g}=8 \times 10^{7} \mathrm{~K}$ and the dashed curve corresponds to the best fit for Model 1 in MB with $T_{g}=2 \times 10^{8} \mathrm{~K}$.

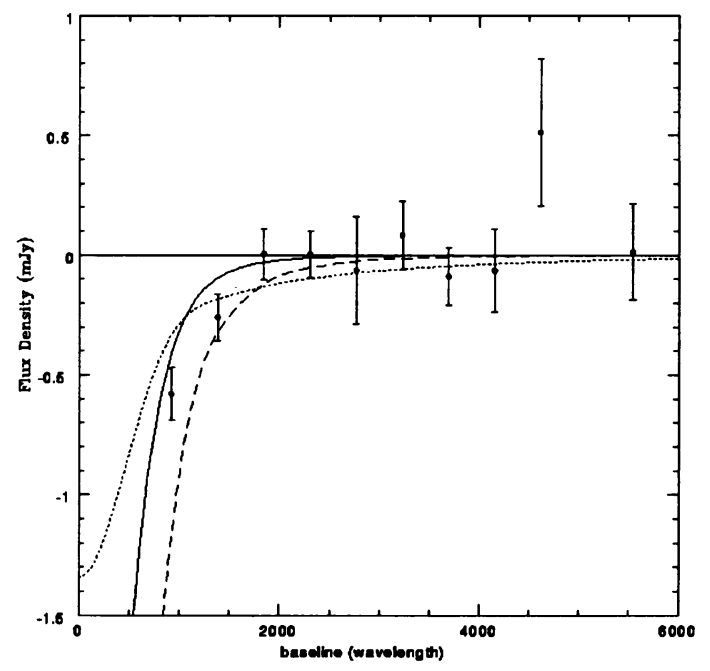

Figure 3. The SZ effect in flux densities versus baseline length. The filled circles are the Ryle data at $15.4 \mathrm{GHz}$ (Jones et al. 1993). The solid curve gives the $\mathrm{SZ}$ effect expected for a cluster potential given in Figure 1 (solid curve) with $T_{g}=8 \times 10^{7} \mathrm{~K}$. The dotted curve is deduced from the cluster parameters that gave the best fit to the X-ray surface brightness for Model 1 of MB at $T_{g}=8 \times 10^{7} \mathrm{~K}$ and the dash curve is deduced from the cluster parameters that gave the best fit to the X-ray surface brightness for Model 1 of $\mathrm{MB}$ at $T_{g}=2 \times 10^{8} \mathrm{~K}$. 
position of the giant arc only constrains the innermost part $(0.08 \mathrm{Mpc})$ of the cluster potential. Given the available data, the cluster potential outside $0.08 \mathrm{Mpc}$ is free to vary and we should be able to vary the outer parts of the cluster potential until it fits the X-ray surface brightness profile while keeping the gas temperature fixed to the observed value. Figure 1 shows an example of such a projected density profile. We assumed that the cluster extends to $6 \mathrm{Mpc}$. The X-ray surface brightness and the SZ effect corresponding to such a potential with $T_{g}=8 \times 10^{7} \mathrm{~K}$ and central electron density $n_{e}(0)=7.3 \times 10^{-3} \mathrm{~cm}^{-3}$ are shown in Figure 2 and 3 respectively. Both the X-ray surface brightness and the SZ effect thus deduced give reasonable fits to the observed data for $H_{0}=50 \mathrm{~km} \mathrm{~s}^{-1} \mathrm{Mpc}^{-1}$. We conclude that, given the data available so far, there is no real conflict between the gravitational lensing constraints from the position of the giant arc and that of the observed data, under the assumption of hydrostatic equilibrium. Ideally, the outer parts of the cluster potential can be determined from a weak lensing analysis, but such analysis requires deep optical data taken in sub-arcsec seeing conditions and was not available until very recently (see Kneib et al. and Kaiser et al. in this conference). It should be noted that the potential shown here is just one of the possible solutions that fits all the data and has is yet to be checked against the amount of shear observed by Kneib et al. and Kaiser et al. (in this proceeding).

\section{Discussion}

In their recent analysis of $\mathrm{A} 2218, \mathrm{MB}$ pointed out that the position of the giant arc in $\mathrm{A} 2218$ is in conflict with the $\mathrm{X}$-ray surface brightness distribution measured by the PSPC and the X-ray temperature measured by the Ginga satellite (see dotted curve in Figure 2). MB assumed that the clusters are spherically symmetric and in hydrostatic equilibrium. They examined two families of parametrized density profiles. The density profiles were constrained by the position of the arc through equation (3). They found that the central mass implied by lensing is a factor of 2-2.5 too large for the gas at the observed temperature to be in hydrostatic equilibrium solely due to thermal pressure support given the family of cluster density profiles. They found that if the gas temperature is raised to 2.5 times larger than the observed temperature, then they could find within their family of density profile one that yields a good fit to the PSPC X-ray surface brightness profile (dashed curve in Figure 2). However, such a model does not fit very well the data for SZ effect (see dashed curve in Figure 3).

The conclusion of MB differs from ours because MB have restricted themselves to certain functional types for the cluster total mass distribution. On the other hand, we should also point out that the validity of the 
potential profile found here in Figure 1 is subject to further analysis. We have not so far examined how the velocity dispersion data can constrain the possible density profiles. However, such constraints of the density profiles by the observed velocity dispersion are limited because of the uncertainties in the galaxy orbits. A more useful constraint comes from the weak lensing analysis which are now available from the works by Kneib et al. and Kaiser et al. (in this proceeding). For example, we can predict the amount of shear that can be produced from the above density distribution and compare that with the results from the weak lensing analysis.

\section{Conclusions}

We found a cluster potential consistent with the "strong" lensing constraint that fits the available data on the SZ effect, the X-ray surface brightness and temperature with $H_{0}=50 \mathrm{~km} \mathrm{~s}^{-1} \mathrm{Mpc}^{-1}$ under the assumption of hydrostatic equilibrium. We have enough information for this cluster to simultaneously determine the shape of the cluster potential and the value of $H_{0}$. With the addition of a temperature profile from ASCA, and a weak lensing analysis of the cluster potential out to $\sim 3 \mathrm{Mpc}$, we can deduce directly the cluster matter distribution, dynamical state and the properties of the ICM. In particular, we can obtain a quantitative measure of the difference in dark and luminous matter density as a function of radius and directly test the consistency of the potential deduced from lensing with that of the X-ray surface brightness and temperature under the assumption of hydrostatic equilibrium. The SZ effect then adds extra information on the value of $H_{0}$ and the clumpiness factor.

\section{References}

Bautz, M., 1995, in New Horizons of X-ray Astronomy, eds. F. Makino \& T. Ohashi, (Universal Academy Press)

Blandford, R. D. \& Kochanek, C. S., 1988, in Dark Matter in the Universe, eds. J. Bachall, T. Piran \& S. Weinberg (Singapore: World Scientific) 133

David, L.P., Slyz, A., Jones, C., Forman, W., Vrtilek, S.D. \& Arnaud, K. A., 1993, Ap. J., 412, 479

Dressler, A., 1976, Ph.D. Thesis, Lick Observatory, University of California, Santa Cruz Jones, M., et al., 1993, Nature, 365, 320

Kaiser, K. \& Squires, G., 1993, Ap. J., 404, 441

Kochanek, C. S., 1992, in Gravitational Lenses, eds. R. Kayser, T. Schramm, L. Nieser (Berlin: Springer) 278

Le Borgne, J. F., Pelló, R. \& Sanahuja, B., 1992, A\&A Suppl., 95, 87

McHardy, I. M., Stewart, G. C., Edge, A. C., Cooke, B., Yamashita, K. \& Hatsukade, I., 1990, MNRAS, 242, 2150

Miralda-Escudé, J. \& Babul, A., 1995, ApJ, 449, 18

Pelló, R., Le Borgne, J. F., Sanahuja, B., Mathez, G. \& Fort, B., 1992, A\&A, 266, 6

Syunyaev, R.A. \& Zel'dovich, Ya.B., 1981, Astrop \& Space Phys Rev, 1, Soviet Scientific Reviews, Section E, 1 